\title{
Food and Nutrition Sciences
}

\section{—Open Special Issues: Public Health Nutrition Initiatives}

\author{
Anthony Fardet \\ National Institute for Agricultural Research, Clermont-Ferrand/Theix Research Center, Saint-Genès-Champanelle, France. \\ Email: anthony.fardet@clermont.inra.fr
}

Received April 26 $6^{\text {th }}, 2013$; revised May 26 $6^{\text {th }}, 2013$; accepted June $3^{\text {rd }}, 2013$

Copyright (C 2013 Anthony Fardet. This is an open access article distributed under the Creative Commons Attribution License, which permits unrestricted use, distribution, and reproduction in any medium, provided the original work is properly cited.

The prevalence of diet-related chronic diseases is dramatically growing each year worldwide oscillating between over- and malnutrition. Thus, in western countries, while cardiovascular diseases and cancers are leading causes of mortality, obesity and type 2 diabetes have now acquired the status of epidemics and are strong risk factors for the development of subsequent more serious chronic diseases. The corresponding human and budget costs for these metabolic diseases are very high. Preventive nutrition is developing within this new context and more and more researches are now focusing on trying to unravel the causal factors responsible for the development of diet-related chronic diseases. In fine, results of these studies have to be transposed in nutritional recommendations for large public. This is not an easy task since, despite the solutions to prevent chronic diseases are known, their prevalence continue to increase.

Thus, it seems today more and more difficult to consume balanced diets, probably due to the generally low level of nutritional education worldwide, the production of highly refined and energy-dense foods by agro-food industry and the high cost of natural and nutrient-dense foods, e.g. fruits and vegetables. Indeed, energy-dense foods are generally cheaper than foods of high nutritional quality, and it is not surprising that obesity prevalence is developing the most among the poorest.

Probably public health nutrition initiatives would have to focus in a near future on nutritional education very early in infancy. In the same time, nutrition research would gain in forwarding from a reductionist towards a more holistic approach, i.e. studying more the health effect of diets combined with the way of living, and avoiding to focus too much on the health effect of isolated food bioactive compounds in relation with a given physiological effect. Preventive nutrition can not be studied using a pharmacological approach: foods are not drugs!

In this special issue, we intend to invite front-line researchers and authors to submit original research and review articles reporting new public health nutrition initiatives, notably those that have succeeded in reducing diet-related chronic disease prevalence. 